\title{
Importância do eletrorretinograma de campo total (Full field ERG) em cães da raça Cocker Spaniel Inglês portadores de catarata ${ }^{1}$
}

\author{
Angélica M.V. Safatle ${ }^{2 *}$, Ana Paula Hvenegaard ${ }^{3}$, Débora Gomes ${ }^{2}$, Daniela C. \\ Leandro $^{3}$, Denise Otsuki ${ }^{4}$ e Ricardo Lisak $^{2}$
}

\begin{abstract}
Safatle A.M.V., Hvenegaard A.P., Gomes D., Leandro D.C., Otsuki D. \& Lisak R. 2010. [Full field electroretinogram in English Cocker Spaniels presenting cataracts.] Importância do eletrorretinograma de campo total (Full field ERG) em cães da raça Cocker Spaniel Inglês portadores de catarata. Pesquisa Veterinária Brasileira 30(2):149-154. Clínica Vetmasters, São Paulo, SP 01415-004, Brazil. E-mail: angsaf@usp.br

Cataracts are one of the most frequent ocular disorders that affects dogs and leads to blindness. In the late stages, lens opacity unables fundoscopy and consequently the diagnosis of retinal degeneration such as progressive retinal atrophy (PRA), which contraindicates surgery. Aiming the return of vision, full field electroretinogram (full field ERG) becomes indispensable before surgery. As English Cocker Spaniels are predisposed to cataracts and retinal degeneration, the objective of this study is the evaluation of the full field ERG records performed in dogs. One hundred thirty-six full field ERGs were recorded from English Cocker Spaniels (62 males and 74 females, with ages ranging from 3 to 15 years old), between September, 2004 and May, 2009. Each examined dog presented cataracts and vision loss before the exam. Diagnosis of retinal degeneration was made based on ERG parameters which were measured from peak to peak amplitude and b-wave implicit time in the 3 different responses (rod response, maximal response and cone response). Amplitude measured in rod, maximal and cone response, as well as the implicit time recorded from dogs that did not present retinal degeneration were respectively: $71.55 \mu \mathrm{V} / 65.15 \mathrm{~ms} ; 149.17 \mu \mathrm{V} / 33.03 \mathrm{~ms}$; and $31.06 \mu \mathrm{V} / 27.90 \mathrm{~ms}$. No response was recorded in $38 \mathrm{dogs}$. Low responses were recorded from the remaining dogs: $12.88 \mu \mathrm{V} / 65.04 \mathrm{~ms}, 24.16 \mu \mathrm{V} / 36.25 \mathrm{~ms}$, and $8.36 \mu \mathrm{V} / 31.38 \mathrm{~ms}$. Results showed that 122 animals $(89.7 \%)$ presented progressive retinal atrophy. Therefore, we conclude that full field ERG must be performed in English Cocker Spaniels presenting cataracts before its removal due to high incidence of retinal degeneration in this breed.
\end{abstract}

INDEX TERMS: Dog, cataracts, retina, full field electroretinogram, progressive retinal atrophy.

RESUMO.- Com o aumento do número de intervenções cirúrgicas para a remoção da catarata em cães, observase a necessidade de exames específicos que devem ser realizados antes da indicação cirúrgica. A catarata é uma das principais causas de cegueira em cães. Nos estágios

\footnotetext{
${ }^{1}$ Recebido em 16 de setembro de 2009.

Aceito para publicação em 7 de dezembro de 2009.

${ }^{2}$ Médico(a) veterinário(a), Clínica Vetmasters, R. Bela Cintra 1500, São

Paulo, SP 01415-004, Brasil. *Autor para correspondência: angsaf@usp.br

${ }^{3}$ Mestranda da Faculdade de Medicina Veterinária e Zootecnia, Universidade de São Paulo (USP), Av. Prof. Dr. Orlando Marques de Paiva 87, Cidade Universitária, São Paulo, SP 05508-270.

${ }^{4}$ Anestesiologia, Faculdade de Medicina, USP, Av. Dr Arnaldo 455, Cerqueira César, São Paulo, SP 01246-903.
}

mais avançados, impossibilita o exame fundoscópico e inviabiliza a observação de alterações retinianas como a atrofia progressiva da retina (APR), degeneração retiniana hereditária de acometimento bilateral que, quando diagnosticada, contra-indica a cirurgia. Com o intuito de reestabelecer a visão, o eletrorretinograma de campo total (Full field ERG) torna-se indispensável na avaliação pré-cirúrgica da remoção da catarata. Como os cães da raça Cocker Spaniel Inglês são predispostos à catarata e a degenerações retinianas, objetivamos neste estudo avaliar as respostas dos ERGs realizados nestes animais. Foram avaliados 136 eletrorretinogramas de cães da raça Cocker Spaniel Inglês (62 machos e 74 fêmeas, com idades entre 3 e 15 anos) no período de Setembro de 2004 a Maio de 2009. Todos os animais apresentavam baixa de visão e catarata durante 0 
exame. O diagnóstico de degeneração retiniana foi baseado nos valores de amplitude pico a pico e tempo de culminação da onda-b nas 3 respostas (resposta escotópica de bastonetes, máxima resposta e resposta fotópica de cones), idade do paciente e estágio de maturação da catarata. Nos cães sem degeneração retiniana, a média da amplitude e do tempo de culminação nas três respostas obtidas foram, respectivamente: $71,55 \mu \mathrm{V} / 65,15 \mathrm{~ms}$; $149,17 \mu \mathrm{V} / 33,03 \mathrm{~ms} ; 31,06 \mu \mathrm{V} / 27,90 \mathrm{~ms}$. Nos cães com degeneração retiniana, 38 animais apresentaram ERG extinto. Dentre os restantes que apresentavam baixas respostas, a média da amplitude e do tempo de culminação nas três respostas obtidas foram, respectivamente: $12,88 \mu \mathrm{V} /$ $65,04 \mathrm{~ms} ; 24,16 \mu \mathrm{V} / 36,25 \mathrm{~ms} ; 8,36 \mu \mathrm{V} / 31,38 \mathrm{~ms}$. Foi observado que em 122 animais $(89,7 \%)$ os exames eram compatíveis com diagnóstico de atrofia progressiva da retina. Frente aos resultados obtidos, conclui-se que os cães da raça Cocker Spaniel Inglês portadores de catarata devem ser submetidos ao eletrorretinograma de campo total antes da remoção cirúrgica devido à alta incidência de degeneração retiniana nesta raça.

TERMOS DE INDEXAÇÃO: Cães, catarata, retina, eletrorretinograma de campo total, atrofia progressiva da retina.

\section{INTRODUÇÃO}

Nos últimos anos, a oftalmologia veterinária vem se tornando uma especialidade cada vez mais difundida e a cirurgia de catarata uma intervenção cada vez mais praticada. A catarata, uma das causas mais frequentes de cegueira em cães (Martin 2005), é definida como qualquer opacidade da lente ou da sua cápsula, independentemente do déficit visual resultante (Davidson \& Nelms 2007). Pode ser classificada de acordo com estágio de desenvolvimento, localização, idade do surgimento, etiologia e consistência (Ofri 2008). Quanto ao estágio de desenvolvimento da catarata, pode-se dividir em quatro diferentes tipos: incipiente, imatura, madura e hipermadura. Esta classificação está relacionada à porcentagem do reflexo tapetal que ela bloqueia. Nas cataratas em estágios mais avançados, a opacidade impossibilita a fundoscopia, inviabilizando a avaliação clínica de alterações evidentes na retina (Davidson \& Nelms 2007, Ofri 2008).

Portanto, com o intuito de restaurar a visão, exames mais específicos como eletrorretinograma de campo total (Full field ERG), devem ser realizados previamente à indicação cirúrgica.

Eletrorretinograma (ERG) é o registro complexo dos potenciais elétricos originários da retina quando o bulbo ocular é estimulado por fonte luminosa. Sua importância se dá por se tratar de um meio diagnóstico objetivo (Tzekov \& Arden 1999), não invasivo (Safatle et al. 2005), capaz de avaliar a função retiniana e detectar precocemente lesões em suas camadas mais externas. Seu registro e análise, denominados eletrorretinografia, são obtidos a partir da superfície da córnea, geralmente por meio de eletrodo de lente de contato. A onda elétrica registrada corresponde à diferença de potencial entre este e um eletrodo de referên- cia, o qual pode estar localizado na própria lente de contato (eletrodo bipolar) ou sobre a pele, desde que próximo ao eletrodo ativo (eletrodo monopolar). Este procedimento é mais sensível que outras técnicas diagnósticas, como a oftalmoscopia, principalmente quando a lesão é sutil ou se encontra em fase inicial (Komaromy et al. 1998).

ERG é frequentemente realizado em pequenos animais, podendo também ser feito em animais de grande porte e exóticos. Em cães, as indicações mais comuns são: avaliação pré-cirúrgica de pacientes com catarata; caracterização de distúrbios que causam cegueira como glaucoma (Rubin 1971), displasia retiniana, retinopatias degenerativas (hereditárias ou não) (Safatle et al. 2005), hipoplasia de nervo óptico e síndrome da degeneração retiniana adquirida subitamente (Narfström et al. 2007). Este exame também pode ser indicado com o intuito de acompanhar a resposta do paciente à terapia instituída (Petersen-Jones et al. 2006).

Dentre as degenerações retinianas hereditárias, a atrofia progressiva da retina (APR) é a mais frequentemente diagnosticada em cães (Petersen-Jones 2005). Quando em fase inicial, as alterações morfológicas se limitam aos segmentos externos dos bastonetes, resultando na desorganização de suas lamelas. Posteriormente, tais alterações degenerativas atingem os segmentos internos e os núcleos dos bastonetes, observando-se ainda a presença de células fagocíticas no espaço sub-retiniano. Nos estágios avançados da doença, as camadas retinianas mais internas são também acometidas (Narfström \& Petersen-Jones 2007).

APR pode ser classificada de diversas formas. Antigamente era classificada em atrofia generalizada ou central da retina, designando a porção acometida. Atualmente, com o desenvolvimento genético e eletrofisiológico, o diagnóstico das alterações retinianas tornou-se mais específico, podendo-se então reclassificá-las de acordo com o fotorreceptor acometido primeiramente, a base molecular ou até mesmo o gene que sofreu mutação, gerando tal alteração (Narfström \& Petersen-Jones 2007). Em cães da raça Cocker Spaniel, APR pode hoje ser denominada como degeneração retiniana progressiva tipo bastonete-cone, referindo-se à célula na qual o processo da doença se inicia. Nestes cães, a doença possui origem hereditária autossômica recessiva (Bjerkas 1990, Parshall et al. 1991) e se caracteriza pela perda progressiva da função retiniana. Animais da raça Cocker Spaniel Inglês desenvolvem manifestações clínicas, como nictalopia, entre 4 e 8 anos de idade. Já os cães da raça Cocker Spaniel Americano apresentam déficit visual em torno de 3 a 5 anos de idade. A APR é geralmente bilateral, tende à simetria (Macmillam \& Lipton 1978, Petersen-Jones 1998, Gelatt 2003, Riis 2005) e não apresenta predisposição sexual (Parshall et al. 1991, Clements et al. 1996, Lin et al. 2002, Bedford 2005). Quando o exame fundoscópico é possível, observa-se atenuação vascular, palidez da papila, hiperreflexia da área tapetal e despigmentação em área não tapetal (Peiffeir Jr. \& Gelatt 1975).

$\mathrm{Na}$ maioria das vezes, a fundoscopia é inviabilizada pela leucocoria (Clements et al. 1996, Safatle et al. 2007). 
Acredita-se que este tipo de catarata resulte da desorganização das fibras do cristalino, supostamente induzida por substâncias tóxicas liberadas pela retina degenerada (Riis 2005). Sendo assim, o ERG torna-se um exame importante, que deve ser realizado antes da indicação cirúrgica para a remoção da catarata em cães.

Devido à alta incidência de catarata e APR em cães da raça Cocker Spaniel Inglês, objetivamos avaliar a função retiniana destes animais por meio do eletrorretinograma de campo total.

\section{MATERIAL E MÉTODOS}

Após realização de anamnese e exame oftalmológico completo incluindo teste de lágrima de Schirmer ${ }^{4}$, teste de fluoresceína ${ }^{5}$, tonometria de aplanação $0^{6}$ e biomicroscopia com lâmpada de fen$\mathrm{da}^{7}$, ERG de campo total foi realizado em 136 cães da raça Cocker Spaniel Inglês (272 olhos), sendo 74 fêmeas e 62 machos, com idades entre 3 e 15 anos (média de 8 anos), que apresentavam catarata e baixa de visão (Fig.1). Os exames foram realizados na Clínica Veterinária Vetmasters, em São Paulo, no período de Setembro de 2004 a Maio de 2009, utilizando o Sistema

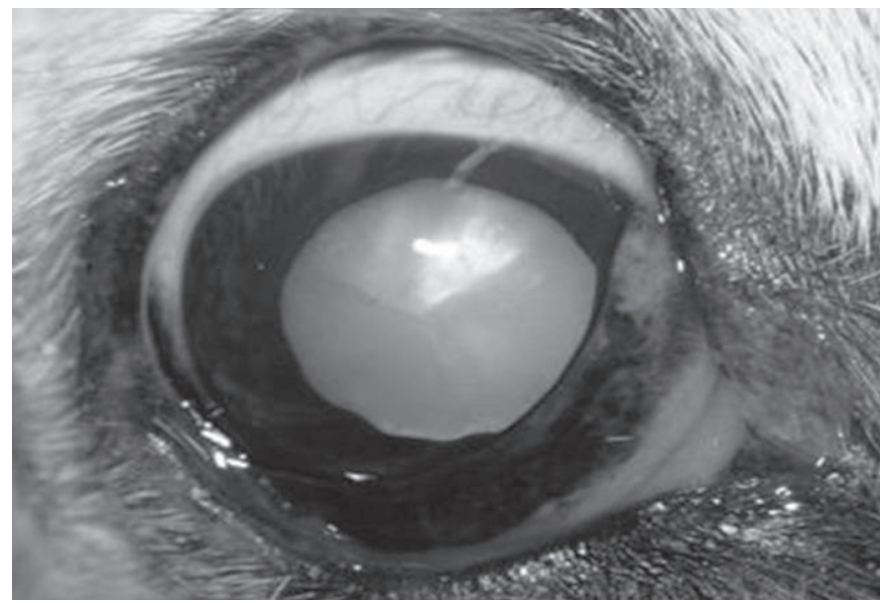

Fig.1. Cão da raça Cocker Spaniel Inglês, fêmea, 10 anos, com catarata hipermadura em olho direito.

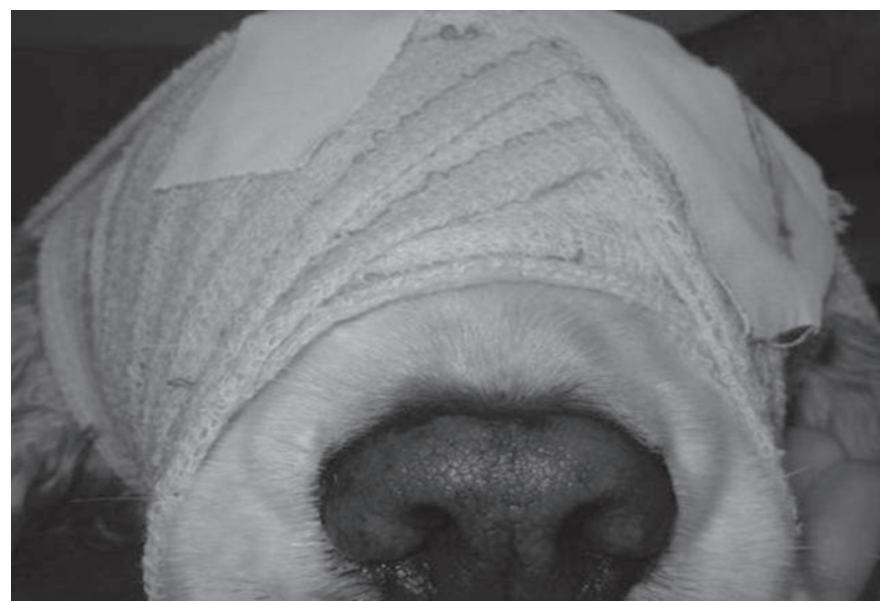

Fig.2. Preparação para o ERG: adaptação ao escuro com bandagem oclusiva em Cocker Spaniel Inglês, macho, 6 anos.
Eletrorretinográfico BPM $200^{8}$. Os animais foram submetidos à sedação e o exame foi conduzido em sala escura. Para tanto, realizou-se administração de sulfato de atropina ${ }^{9}$ (0,044mg/kg/ peso, SC) e foi instilado em ambos os olhos colírio de tropicamida $1 \%{ }^{10}$. A adaptação ao escuro foi feita com uso de bandagem oclusiva ${ }^{11}$ (Fig.2) e teve duração de 30 minutos. Para sedação, foram administrados: cloridrato de xilazina ${ }^{12}(0,2 \mathrm{mg} / \mathrm{kg} / \mathrm{peso}, \mathrm{IM})$ e butorfanol ${ }^{13}(0,2 \mathrm{mg} / \mathrm{kg} /$ peso, IM). Após a instilação de colírio anestésico ${ }^{14}$, posicionou-se o eletrodo monopolar ERG-jet ${ }^{15}$ sobre a córnea, uma vez preenchido com metilcelulose $2 \%{ }^{16}$, a fim de oferecer melhor contato elétrico e proteger a superfície corneal. O eletrodo de referência de cúpula de ouro ${ }^{17}$ preenchido com creme eletrolítico ${ }^{18}$ foi posicionado no canto temporal do olho, após tricotomia e higienização da pele (Fig.3). O eletrodo-terra, similar ao de referência, foi colocado na face interna do pavilhão auricular tricotomizado, após preenchê-lo também com creme eletrolítico. Com os eletrodos devidamente colocados, posicionou-se o animal em decúbito lateral direito para a realização da fase escotópica em olho direito e, posteriormente, em olho esquerdo. Após 10 minutos de adaptação ao claro, iniciou-se a fase fotópica do exame em olho esquerdo, finalizando o exame em olho direito. Observou-se, com frequência, a centralização dos olhos. Caso estivessem rotacionados ou apresentassem protrusão da terceira pálpebra, estimulava-se o paciente com sinais sonoros. A primeira resposta registrada foi a resposta de bastonetes, registrada 30 minutos após a adaptação ao escuro, empregando estímulo de luz branca com sua intensidade máxima atenuada em 2.0 unidades logarítmicas, utilizando filtro de densidade neutra e intervalo de 2 segundos entre os estímulos. A segunda resposta, denominada resposta escotópica máxima, foi obtida após estímulos de alta intensidade, contendo tanto as respostas de cones como dos bastonetes. O estímulo foi realizado após adaptação prévia ao escuro, com intervalo de 10 segundos interestímulos. Após adap-

4 Teste de Schirmer. Ophthalmos Indústria Farmacêutica, Rua Nhandirobas 471, , São Paulo, SP 04349-030.

${ }^{5}$ Colírio de fluoresceína 1\%. Ophthalmos Indústria Farmacêutica, Rua Nhandirobas 471, São Paulo, SP 04349-030.

6 Tono-pen.XL Mentor, CA, EUA.

7 SL-15. Kowa Optimed, Incorporation, Torrance, CA, EUA.

${ }^{8}$ BPM200 Electrodiagnostic System. RetinoGraphics Inc., 9 Dock Road, Norwalk, CT, EUA.

${ }^{9}$ Atropion. Ariston Indústrias Químicas e Farmacêuticas Ltda, Rua Adherbal Stresser 84, Jd. Arpoador, São Paulo, SP 05566-000.

${ }^{10}$ Mydriacyl. Alcon Laboratórios do Brasil Ltda., Av. Nossa Senhora Assunção 736, VI. Butantã, São Paulo, SP 05359-001.

${ }^{11}$ Atadura de Crepon Neve. Neve Indústria e Comércio de Produtos Cirúrgicos Ltda, Rua Júlio Parigot 353, VI. Antonieta, São Paulo, SP 03478-007.

12 Xilazin. Syntec do Brasil Ltda, Rua Soluções do Lar 105, Cotia, SP 06716-020.

13 Torbugesic. Fort Dodge Saúde Animal Ltda, Rua Luiz Fernando Rodriguez 1701, VI. Boa Vista, Campinas, SP 13065-858.

${ }^{14}$ Anestalcon. Alcon Laboratórios do Brasil Ltda, Avenida Nossa Senhora Assunção 736, VI. Butantã, São Paulo, SP 05359-001.

15 ERG-jet. Fabrinal SA, 42 Rue de la Tuilerie, La Chaux-de-Fonds, Suiça.

${ }^{16}$ Metilcelulose 2\%. Ophthalmos Indústria Farmacêutica, Rua Nhandirobas 471, São Paulo, SP 04349-030.

17 Grass Technologies, Astro-Med Inc., 600 East Greenwich Avenue, West Warwick, RI, EUA.

18 Pasta Disk-Fix. Meditron Eletromedicina Ltda, R. Alexandre Finta 99, Santo Amaro, São Paulo, SP 05850-090. 


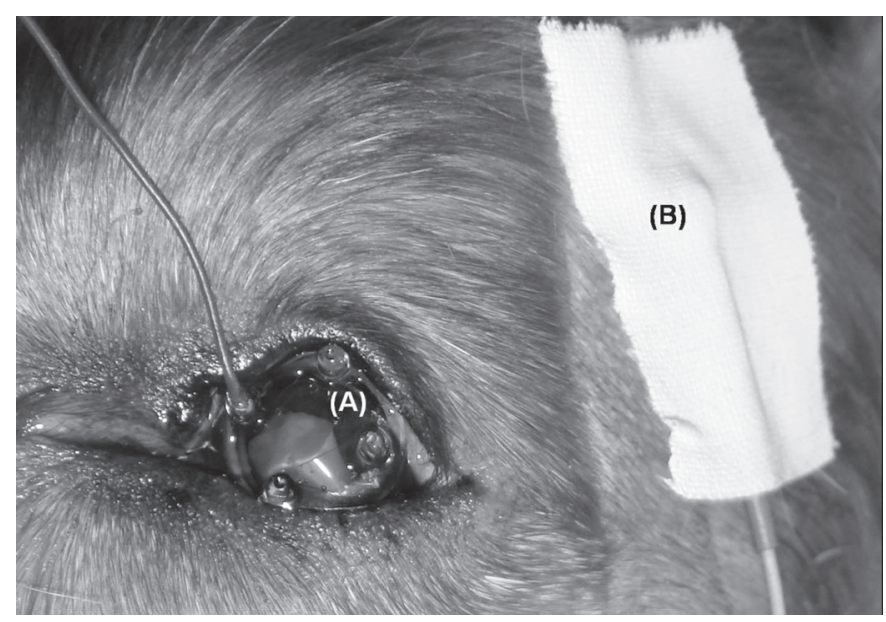

Fig.3. Posicionamento dos eletrodos em Cocker Spaniel Inglês, macho, 4 anos de idade: (A) eletrodo monopolar ERG-jet preenchido com metilcelulose $2 \%$ posicionado sobre a córnea; (B) eletrodo de referência de cúpula de ouro (GRASS) preenchido com creme eletrolítico posicionado no canto temporal do olho, após tricotomia e higienização da pele, fixado com auxílio de esparadrapo.
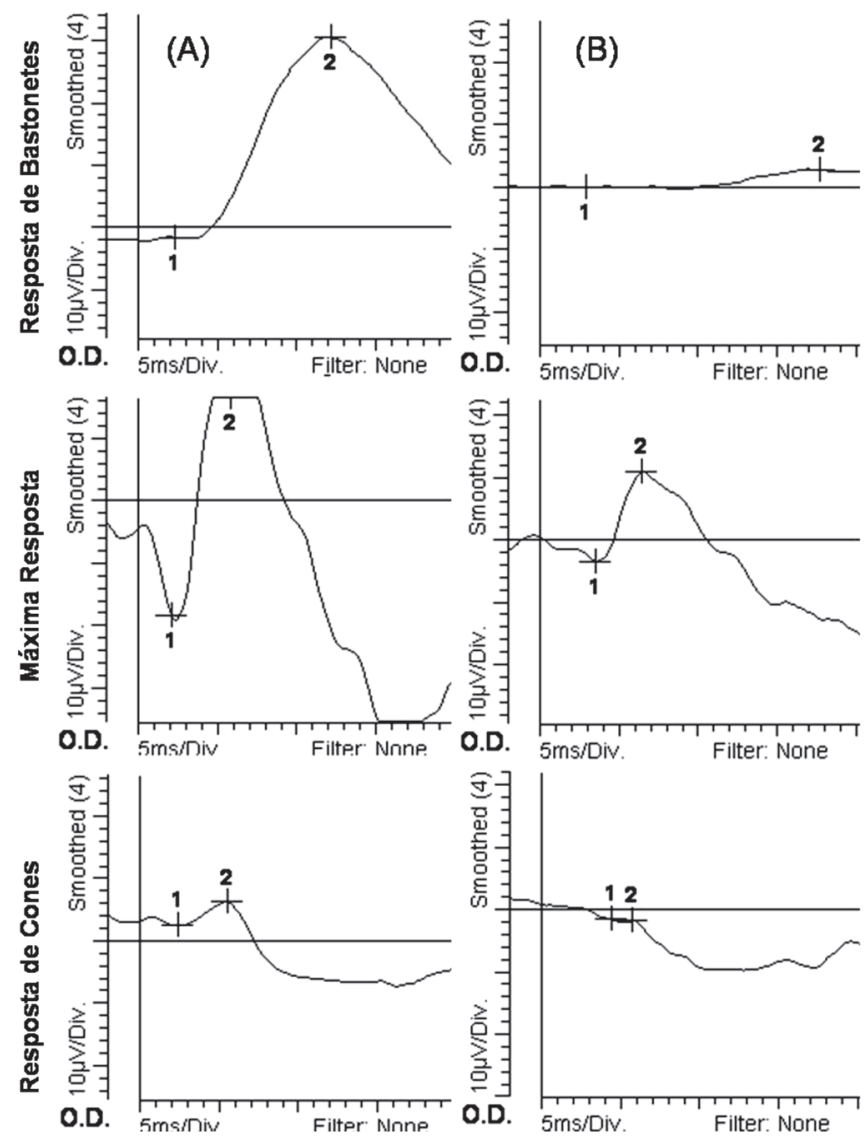

tação ao claro durante 10 minutos, registrou-se a terceira resposta, denominada resposta de cones. Nesta resposta, isolou-se a atividade dos cones, utilizando estímulos luminosos de intensidade máxima, com intervalo de 1 segundo entre eles. Ao término do exame, mensurou-se a amplitude pico a pico (pico da ondaa até o pico da onda-b) e o tempo de culminação da onda-b nas três respostas acima descritas de cada paciente (Fig.4).

Os valores de amplitude e tempo de culminação da onda b das três respostas obtidas serão apresentados de forma descritiva. Respostas extintas, observadas em alguns animais portadores de degeneração retiniana, foram desconsideradas para a análise descritiva do grupo. A distribuição de fêmeas e machos foi avaliada entre os grupos por meio do teste de quiquadrado. A idade dos animais nos diferentes grupos foi comparada por meio do teste de Kruskal-Wallis. A amplitude e o tempo de culminação da onda $b$ das três respostas foram comparados entre os três grupos por meio do teste de Kruskal-Wallis, seguido de teste de Dunn quando adequado.

\section{RESULTADOS}

O diagnóstico de degeneração retiniana foi baseado nos valores de amplitude pico a pico e tempo de culminação da onda-b nas 3 respostas (resposta escotópica de bastonetes,

Fig.4. Modelo de eletrorretinografia de campo total utilizando o Sistema Eletrorretinográfico BPM 200. (A) Olho direito de Cocker Spaniel Inglês, fêmea, 11 anos, com catarata madura bilateral sem alteração retiniana. (B) Olho direito de Cocker Spaniel Inglês, macho, 12 anos, com catarata madura bilateral com alteração retiniana e ERG com respostas atenuadas. (C) Olho direito de Cocker Spaniel Inglês, macho, 10 anos, com catarata madura bilateral com alteração retiniana e ERG extinto. 
máxima resposta e resposta fotópica de cones), idade do paciente e estágio de maturação da catarata.

Dentre os 136 animais incluídos neste estudo, 14 (10,30\%) cães não apresentavam degeneração retiniana, sendo 8 fêmeas e 6 machos, com idades entre 3 e 15 anos (média de 7,6 anos, com desvio padrão de 3,8 anos). Nestes, a média da amplitude e do tempo de culminação nas três respostas obtidas foram, respectivamente: $71,55 \mu \mathrm{V}$ (desvio padrão igual a $36,02 \mu \mathrm{V}$ ) e $65,15 \mathrm{~ms}$ (desvio padrão igual a $5,73 \mathrm{~ms}$ ); $149,17 \mu \mathrm{V}$ (desvio padrão igual a 49,56 $\mu \mathrm{V}$ ) e 33,03ms (desvio padrão igual a 4,19ms); $31,06 \mu \mathrm{V}$ (desvio padrão igual a $17,23 \mu \mathrm{V}$ ) e $27,90 \mathrm{~ms}$ (desvio padrão igual a $2,76 \mathrm{~ms}$ ).

Dentre os $122(89,70 \%)$ cães portadores de degeneração retiniana, $38(27,94 \%)$ animais apresentaram ERG extinto. Este grupo era constituído por 18 fêmeas e 20 machos, com idades entre 4 e 11 anos (média de 7,9 anos, com desvio padrão de 1,7 anos). Os $84(61,76 \%)$ cães restantes, 48 fêmeas e 36 machos, com idades entre 4 e 14 anos (média de 8,7 anos, com desvio padrão de 1,8 anos), apresentaram respostas de baixa amplitude. A média da amplitude e do tempo de culminação nas três respostas obtidas foram, respectivamente: $12,88 \mu \mathrm{V}$ (desvio padrão igual a 13,22 $\mu \mathrm{V}$ ) e 65,04ms (desvio padrão igual a 9,92ms); $24,16 \mu \mathrm{V}$ (desvio padrão igual a $28,98 \mu \mathrm{V}$ ) e $36,25 \mathrm{~ms}$ (desvio padrão igual a $4,55 \mathrm{~ms}$ ); $8,36 \mu \mathrm{V}$ (desvio padrão igual a $9,48 \mu \mathrm{V}$ ) e 31,38ms (desvio padrão igual a 5,05ms) (Quadro 1).

A distribuição de fêmeas e machos é igual entre os grupos (teste de qui-quadrado 1,055 com 2 graus de liberdade, $P=0,590)$. A idade entre os diferentes grupos não apresenta diferença estatística (teste de Kruskal-Wallis, $p=0,08$ ).

\section{DISCUSSÃO}

A evolução da oftalmologia veterinária trouxe consigo exames específicos para o auxílio diagnóstico de doenças retinianas, como o ERG de campo total, garantindo desta forma a indicação correta da remoção cirúrgica da catarata.
ERG é um exame objetivo (Tzekov \& Arden 1999) e eficaz no diagnóstico de degeneração retiniana, uma vez que este procedimento é mais sensível que outras técnicas diagnósticas como oftalmoscopia, principalmente quando a lesão é sutil, está em fase inicial (Komaromy et al. 1998) ou quando o animal apresenta opacidade de meios como catarata.

Para a realização do exame, necessita-se da cooperação do paciente, que deve permanecer imóvel (Narfström 2006) e apresentar midríase máxima, com o intuito de estimular amplamente a retina (Birch 1989). O protocolo de realização do exame foi o mesmo proposto por outros autores e permitiu o adequado posicionamento do animal e a obtenção de registros confiáveis e com baixo ruído. (Safatle et al. 2006).

A realização do ERG unilateral ou bilateral simultâneo depende da espécie, do aparelho e da indicação para o exame (Narfström 2006). Neste estudo, os dois olhos foram estimulados individualmente.

A interpretação do ERG deve ser feita levando-se em consideração: opacidade de meios, eletrodos, aparelho eletrorretinográfico, protocolo de estimulação, espécie, raça e idade (Narfström 2006, Petersen-Jones et al. 2006). No presente estudo, todos os animais eram cães da raça Cocker Spaniel Inglês e apresentavam catarata importante em ambos os olhos. Outros autores também observaram uma elevada incidência de catarata em cães desta raça, de acometimento geralmente bilateral, cuja formação pode ser secundária à degeneração retiniana (Macmillam \& Lipton 1978, Petersen-Jones 1998, Gelatt 2003, Riis 2005).

Apesar de também haver alta incidência de degeneração retiniana em cães da raça Cocker Spaniel Americano (Narfström \& Petersen-Jones 2007), estes não foram incluídos no presente estudo devido ao reduzido número de pacientes desta raça submetidos ao ERG neste período.

Como a degeneração retiniana foi diagnosticada em todas as faixas etárias, demonstrou-se que, embora a ida-

\begin{tabular}{|c|c|c|c|}
\hline & \multicolumn{3}{|c|}{ Animais (Grupos) } \\
\hline & \multirow[t]{2}{*}{ Normais } & \multicolumn{2}{|c|}{ Degeneração retiniana } \\
\hline & & ERG não extinto & ERG extinto \\
\hline Número de animais (porcentagem) & $14(10,3 \%)$ & $84(61,76 \%)$ & $38(27,94 \%)$ \\
\hline Idade em anos - média (desvio padrão) & $7,6(3,8)$ & $8,7(1,8)$ & $7,9(1,7)$ \\
\hline $\begin{array}{l}\text { Amplitude pico a pico em } \mu \mathrm{V} \\
\text { - média (desvio padrão) }\end{array}$ & & & \\
\hline Resposta de Bastonetes & $71,55^{\mathrm{ab}}(36,02)$ & $12,88^{\mathrm{a}}(13,22)$ & $0,00(0,00)$ \\
\hline Máxima Resposta & $149,17^{\mathrm{ab}}(49,56)$ & $24,16^{\mathrm{a}}(28,98)$ & $0,00(0,00)$ \\
\hline Resposta de Cones & $31,06^{\mathrm{ab}}(17,23)$ & $8,36^{a}(9,48)$ & $0,00(0,00)$ \\
\hline $\begin{array}{l}\text { Tempo de culminação da onda b em } \mathrm{m} \\
\text { - média (desvio padrão) }\end{array}$ & & & \\
\hline Resposta de Bastonetes & $65,15^{a}(5,73)$ & $65,04^{a}(9,92)$ & $0,00(0,00)$ \\
\hline Máxima Resposta & $33,03^{a b}(4,19)$ & $36,25^{a}(4,55)$ & $0,00(0,00)$ \\
\hline Resposta de Cones & $27,90^{\mathrm{ab}}(2,76)$ & $31,38^{a}(5,05)$ & $0,00(0,00)$ \\
\hline
\end{tabular}

a Diferente do grupo ERG extinto: $p<0,05$.

b Diferente do grupo ERG não extinto: $p<0,05$. 
de influencie a resposta do ERG (Narfström 2006), esta não deve ser considerada uma variável significativa no diagnóstico da degeneração. Observou-se também que não houve predisposição sexual para incidência da degeneração, o que já foi observado em outros estudos (Parshall et al. 1991, Clements et al. 1996, Lin et al. 2002, Bedford 2005).

Com os resultados obtidos, pode-se observar diferença importante entre os valores eletrorretinográficos, possibilitando diagnosticar animais com e sem alterações retinianas.

A atrofia progressiva da retina foi confirmada em 122 cães. Destes, 84 animais apresentaram baixa amplitude e atraso no tempo de culminação das três respostas testadas. Nos 38 cães restantes, observou-se ausência completa das ondas-a e b, ou seja, ERG extinto (zero).

Sugere-se que o ultrassom ocular também seja realizado, caso se observe assimetria importante dos valores obtidos nos registros entre os olhos, a fim de se excluir o descolamento antigo e total da retina (Gonçalves et al. 2000). Neste estudo, o ERG foi realizado em ambos os olhos e as respostas tenderam à simetria. Se ainda assim, as respostas forem simétricas, porém extintas, devese excluir o diagnóstico da síndrome da degeneração retiniana adquirida subitamente. Diferentemente do PRA, esta se manifesta causando cegueira repentina e acometendo principalmente fêmeas castradas, de meia idade, com histórico de poliúria, polidipsia, polifagia e ganho de peso recente (Cullen \& Grahn 2002). Tais alterações acima descritas foram observadas durante a anamnese e exame clínico em dois cães da raça Cocker Spaniel Inglês. Entretanto, estes animais não foram incluídos no estudo, uma vez que não apresentavam catarata.

\section{CONCLUSÕES}

Concluímos que a atrofia progressiva da retina e a catarata são doenças frequentes nos cães da raça Cocker Spaniel Inglês. Nesses casos, sugere-se que, antes de propor procedimento cirúrgico para a remoção da catarata, sejam realizados exames complementares como o eletrorretinograma de campo total, visando avaliar a função retiniana.

Como a degeneração possui origem genética, sugere-se também que estes animais sejam excluídos do programa de reprodução, a fim de evitar sua propagação.

\section{REFERÊNCIAS}

Bedford P.G.C. 2005. Progressive retinal atrophy in dogs. Vet. Rec. 156(4): 124.

Birch D.G. 1989. Clinical Electroretinography. Ophthalmol. Clin. North Am. 2(3):469-497.

Bjerkas E. 1990. Generalised progressive retinal atrophy in the English setter in Norway. Vet. Rec. 126(9):217.

Clements P.J.M., Sargan D.R., Gould D.J. \& Petersen-Jones S.M. 1996. Recent advances in understanding the spectrum of canine generalised progressive retinal atrophy. J. Small Anim. Pract. 37(4):155-162.

Cullen C.L. \& Grahn B.H. 2002. What are your clinical diagnosis, lesion localization, and differential diagnoses? Can. Vet. J. 43(9):729-730.
Davidson M.G. \& Nelms S.R. 2007. Diseases of the canine lens and cataract formation, p.859-887. In: Gelatt K.N. (Ed.), Veterinary Ophthalmology. $4^{\text {th }}$ ed. Blackwell Publishing, Ames, lowa.

Gelatt K.N. 2003. Manual de Oftalmologia Veterinária. Manole, São Paulo, p.227-252.

Gonçalves E.R., Fernandes M.L., Takahashi W.Y. \& Suzuki H. 2000. Eletrofisiologia ocular, p.185-225. In: Conselho Brasileiro de Oftalmologia, Sociedade Brasileira de Retina e Vítreo (Ed.), Retina e Vítreo: clínica e cirurgia. Roca, São Paulo.

Komaromy A.M., Smith P.J. \& Brooks D.E. 1998. Electroretinography in dogs and cats. Part I. Retinal morphology and physiology. Compend. Contin. Educ. Vet. 20:343-354.

Lin C.T., Gould D.J., Petersen-Jones S.M. \& Sargan D.R. 2002. Canine inherited retinal degenerations: Update on molecular genetics research and its clinical application. J. Small Anim. Pract. 43(10):426-432.

MacMillam A.D. \& Lipton D.E. 1978. Heritability of multifocal retinal dysplasia in American Cocker Spaniels. J. Am. Vet. Med. Assoc. 172(5):568-572.

Martin C.L. 2005. Vitreous and ocular fundus, p.401-470. In: Ibid. (Ed.), Ophthalmic Disease in Veterinary Medicine. Manson Publishing, London.

Narfström K. 2006. Electroretinographic testing in larger animals, p.923933. In: Heckenlively J.R. \& Arden G. B. (Eds), Principles and Practice of Clinical Electrophysiology of Vision. The Mit Press, Cambridge.

Narfström K. \& Petersen-Jones S. 2007. Diseases of the canine ocular fundus, p.944-1058. In: Gelatt K.N. (Ed.), Veterinary Ophthalmology. Backwell Publishing, Ames, lowa.

Narfström K., Wrigstad A., Ekesten B. \& Bergss L.A. 2007. Neuronal ceroid lipofuscinosis: Clinical and morphologic findings in nine affected Polish Owczarek Nizinny (PON) dogs. Vet. Ophthalmol. 10(2):111-120.

Ofri R. 2008. Lens, p.258-276. In: Maggs D.J., Miller P.E. \& Ofri R.I. (Eds), Slatter's Fundamentals of Veterinary Ophthalmology. $4^{\text {th }}$ ed. Saunders Elsevier, St Louis.

Parshall C.J., Wyman M., Nitroy S., Acland G. \& Aguirre G. 1991. Photoreceptor dysplasia: An inherited progressive retinal atrophy of Miniature Schnauzer dogs. Progr. Vet. Comp. Ophthalmol. 1(3):187-203.

Peiffeir Jr R.L. \& Gelatt K.N. 1975. Progressive retinal atrophy in two atypical breeds of dogs. Vet. Med. Small Anim. Clin. 70(12):1476-1478.

Petersen-Jones S.M. 1998. A review of research to elucidate the causes of the generalized progressive retinal atrophies. Vet. J. 155:5-18.

Petersen-Jones S. 2005. Advances in the molecular understanding of canine retinal diseases. J. Small Anim. Pract. 46(8):371-380.

Petersen-Jones S., Tuntivanich N., Montiani-Ferreira F. \& Khan N.W. 2006. Electroretinograms of dog and chicken, p.911-921. In: Heckenlively J.R. \& Arden G.B. (Eds), Principles and Practice of Clinical Electrophysiology of Vision. $2^{\text {nd }}$ ed. Cambridge, UK.

Riis R.C. 2005. Degenerações da retina, p.294-305. In: Ibid. (Ed.), Segredos em Oftalmologia de Pequenos Animais. Artmed, Porto Alegre.

Rubin L.F. 1971. Clinical features of hemeralopia in adult Alaskan Malamute. J. Am. Vet. Med. Assoc. 158(10): 696-1698.

Safatle A.M.V., Salomão S., Berezovsky A., Sacai P., Fantoni D., Yasbek K. \& Barros P.S.M. 2005. Retinal degeneration in a Pit Bull dog: Electroretinographic findings. Archs Vet. Sci. 10(2):119-124.

Safatle A.M.V., Barrio M.A.D., Lisak R., Galego M.P. \& Kahvegian M. 2006. Protocolo de Eletrorretinograma em cães. XXVII Congresso Brasileiro da Anclivepa, Vitória, p.82.

Safatle A.M.V., Gomes D., Otuski D.A., Shimamura G.M., Hvenegaard A.P.F.A. \& Lisak R. 2007. Estudo retrospectivo dos resultados do eletrorretinograma de campo total em cães da raça Cocker portadores de catarata. In: Anais $7^{\circ}$ Congresso Paulista de Clínicos Veterinários de Pequenos Animais, Anclivepa, São Paulo, p.228-230.

Tzecov R. \& Arden G.B. 1999. The electroretinogram in diabetic retinopathy. Surv. Ophthalmol. 44(1):53-60. 